\title{
Determination of gold in alloys via potentiometric titration; an alternative to the fire assay
}

\author{
S. Caporali*, S. Bellandi, M. Innocenti, O. Lopilato, L. Romualdi and G. Pezzatini \\ www.goldbulletin.org
}

\begin{abstract}
In this paper we report the results of a deep investigation of gold title in jewellery alloys by means of potentiometric titration. After reviewing the suitable reactions for such purpose we focused on the most profitable one, which involves the use of hexadecylpyridinium chloride (CPC). This cation gives rise to selective and quantitative precipitation of $\mathrm{AuCl}_{4}:$.We completely revised a method previously proposed by W.S. Selig in the 80's, by improving the sampling preparation and by optimizing the titration steps. Then, the new proposed method was tested on a large number of different alloys, commonly used for goldsmithery purposes. The obtained gold titles were compared with the data achieved by fire assay, giving rise to a very good agreement. At the light of these results, the present method can be rightfully considered a cheaper and "greener" alternative to the traditional one.
\end{abstract}

\footnotetext{
University of Florence, Department of Chemistry,

Via della Lastruccia 3, 50019 Sesto Fiorentino, Italy

* Corresponding author: Fax +39055 3473120

E-mail: stefano.caporali@unifi.it
}

Keywords: gold alloys, potentiometric titration, surface modified electrodes, cethylpyridinium chloride, cupellation

\section{Introduction}

The determination of gold title in jewellery-used alloys remains one of the most challenging tasks in analytical chemistry and constitutes a demanding chore owing to the large number of metals used in goldsmithery and the wide compositional variety ranging from a few up to more than $90 \% \mathrm{~W}$. Furthermore, due to gold's high economic value, its quantification must be carried out with high accuracy. The problem has been satisfactorily solved by the so-called cupellation, or lead fire-assay. In fact the scientific assessment of this method, whose principle takes root in the ancient history, was accomplished in the first half of the last century [1]. Nevertheless, the cupellation, owing to its high precision and accuracy, is still the preferred method for determination of gold in jewellery handcrafts $[2,3]$.

The classical lead assay method is based on melting gold-containing alloys, mixed with high purity lead and silver, in special crucibles made of $\mathrm{MgO}$ (or traditionally bone ash), called cupellas (so the name cupellation for this analysis). The porous material of the crucible retains non-noble metal oxides, whereas gold and silver form a bead that is laminated, immersed in $\mathrm{HNO}_{3}$ solution in order to dissolve silver, and finally weighed. The method at issue is therefore classified as a gravimetric one, and its force relies in its exceptional precision and accuracy. These can be expressed as a relative standard deviation (rsd) of $\pm 0.5 \%$. On this subject F.E. Beamish in his first monograph [2] reported that to his knowledge the lead fire assay, if properly employed, never failed, and this statement is still true. However the method suffers from several drawbacks such as: (1) a quite expensive and cumbersome laboratory equipment and procedures exclusively devoted to this specific determination; (2) a huge waste of energy unavoidable in keeping the furnace at the operating 
temperature $\left(1100^{\circ} \mathrm{C}\right)$; (3) releasing of high amounts of vapour lead oxide, that is recognized to be an important environmental pollutant; (4) the content of gold must be roughly known in order to achieve the correct silver-to-gold ratio $(4: 1$, also called inquartation); (5) a relevant quantity of expensive (silver) and hazardous $\left(\mathrm{HNO}_{3}\right)$ chemicals are consumed; (6) the results are strongly dependent on the experience and skill of the assayer.

Therefore, especially in recent decades, many instrumental methods of analysis such as Atomic Absorption Spectrometry (AAS), Inductively Coupled Plasma (ICP) [4], neutron activation analysis (NAA) [5], X-ray fluorescence spectrometry (XRF) [6,7], laser induced breakdown spectroscopy (LIBS) $[8,9]$ have been proposed for gold determination. However, all these techniques require the attention of a highly trained and skilled operator, are strongly affected by matrix effects or are surface sensitive. In addition, some of these methods are devoted to the determination of trace elements and are hardly applicable to the accurate determination of the main components of goldsmithery handcrafts. Recently, Knudsen Effusion Mass Spectrometry (KEMS) was applied to gold assay with good results [10] but the cost of the apparatus and the required assayer's skill, make this technique out of many small-firms budget.

Looking for simple and cheap techniques that can compete with lead fire assay as concerns precision and accuracy, we investigated within the electrochemical titration methods. In an extended series of papers [11-13] Selig reported experiments on many types of potentiometric titrations, by using surface-modified indicator electrodes and quaternary ammonium halides as titrants. The titration of gold $\left(\mathrm{AuCl}_{4}^{-}\right.$anion) via formation of an insoluble ionic couple with hexadecylpyridinium cation (whose chloride salt is also called cethylpyridinium chloride or CPC) resulted particularly suitable to our purpose. The course of the reaction was potentiometrically monitored using a selective surface-modified electrode to fix accurately the equivalent point. By combining the relative selectivity of both the precipitation of $\mathrm{AuCl}_{4}^{-}$by CPC and the adsorption of this one on the electrode surface, the titration method suffers from very little interferences.

In the present paper, after a short review of the reactions potentially suitable for gold potentiometric titration, we report further investigations and deep improvements of Selig's method. They concern, respectively: (1) the best way of quantitatively dissolving the gold-alloys samples to be subjected to titration; (2) the use of more flexible and efficient titration apparatus than available to Selig; (3) the right evaluation of the titration curves; (4) the optimization of the amounts of samples and of the total time consumed for each analysis.

Furthermore, in order to confirm the complete set-up procedure, an extensive investigation on the stability of the titrant solution and of graphite electrodes, as well as tests on various types of goldsmithery alloys, have been performed. Finally, the data obtained from the different series of titrations were compared with lead fire-assay results with unprecedented agreement.

\section{Review of the reactions potentially viable for potentiometric titration of gold}

In theory, there are many reactions suitable for quantitative determination of gold in aqueous solutions. These can be subdivided between redox and precipitation reactions. In the first group, several inorganic reducing agents such as $\mathrm{Fe}(\mathrm{II}), \mathrm{Ti}(\mathrm{III})$, $\mathrm{Sn}(\mathrm{II}), \mathrm{Cr}(\mathrm{III}), \mathrm{Cu}(\mathrm{I})$, arsenite and iodide have been proposed [14]. They are more or less specifically designed to reduce only $\mathrm{Au}(\mathrm{III})$ to $\mathrm{Au}(\mathrm{I})$ or $\mathrm{Au}(0)$. Among this group of reactants, iodide was the most investigated since K. Someya proposed its analytical application in 1930 [15]. The method is based on the reduction of $A u(I I I)$ to $A u(I)$ giving rise to the formation of $\mathrm{I}_{2}$ :

$\mathrm{AuCl}_{4}^{-}+2 \mathrm{I}^{-} \mathrm{AuCl}_{2}^{-}+\mathrm{I}_{2}+2 \mathrm{Cl}^{-}$

Even though several variations of the method have been proposed, the end point is determined potentiometrically using the back-titration of the liberated iodine. However, this method is affected by the drawback of the indirect determination, and the results are not so accurate as the ones obtained by the fire assay.

Direct titrations do not suffer from such drawback, allowing higher accuracy. With this intent, several organic reagents such as hydroquinone, ascorbic acid, o-dianisidine and hydrazine have been proposed and investigated. Among them, hydroquinone seemed to be the most profitable. The method, as proposed by W.B. Pollard [16], consists in the precipitation of metallic gold from chloroauric solutions according to the following scheme: 


$$
2 \mathrm{AuCl}_{4}{ }^{-}+3 \mathrm{C}_{6} \mathrm{H}_{6} \mathrm{O}_{2} \quad 2 \mathrm{Au}+3 \mathrm{C}_{6} \mathrm{H}_{4} \mathrm{O}_{2}+8 \mathrm{Cl}^{-}+6 \mathrm{H}^{+}
$$

The reaction is quantitative and rapid enough to be analytically applicable. Furthermore, Beamish and co-workers [17] state that it is subjected to very low interferences from $\mathrm{Pt}$ and platinum group metals (PGM). However, the results are less precise and accurate than fire assay. The back-titration of the excess of hydroquinone by cerium sulphate was proposed [18] in order to improve the analytical precision, but the results were not satisfactory. The same considerations hold for ascorbic acid, o-dianisidine and hydrazine. Therefore, the use of CPC seems to be the only one worthy of further investigation, except for the really unusual presence of Pt in gold alloys used in jewelery.

\section{Experimental}

\subsection{Reagents}

Chemicals were analytically pure from Merck or Sigma Aldrich. Water, demineralized by ion-exchange resins, was further purified by two distillations, the second of which was from alkaline permanganate. Standard solutions of $\mathrm{Au}(\mathrm{III})$ were obtained from pieces of $99.99 \%$ pure gold wire $(0.5 \mathrm{~mm}$ diameter) supplied by Platecxis (France), by weighing them to $\pm 0.01 \mathrm{mg}$ and dissolving as described in the discussion section.

Titrant (CPC) solutions were prepared monthly and stored in dark glass bottles: the exact title was determined against standard solutions of gold prior to analyze real samples and its value was found to vary no more than $\pm 0.1 \%$. Commercial grade goldsmithery alloys, whose titles range from 385/1000 up to $917 / 1000$, were supplied by commercial firms together with their gold titles determined by cupellation.

\subsection{Apparatus and electrodes}

The sample dissolution was carried out by using a microwave acid digestion bomb (Parr 4782) constituted by a self-sealing PTFE vessel held in a chemically inert micro-wave transparent jacket. Details about this apparatus and its use can be found elsewhere $[19,20]$ while the operating procedure will be described in section 4.1.

Double wall thermostatted titration vessels were used to carry out the titration at $23( \pm 0.5)^{\circ} \mathrm{C}$ following Selig's suggestions [13].
$\mathrm{Ag} / \mathrm{AgCl} / 3 \mathrm{M} \mathrm{KCl}$ reference electrode was connected to the cell through a portion of $0.1 \mathrm{M} \mathrm{HCl}$ solution and a double porous diaphragm. The indicator electrodes consisted of spectroscopic graphite rods covered by a sensitive organic film of diocthylphtalate (DOP) dispersed in polivnyilchloride (PVC). These electrodes were prepared in our lab by optimizing the procedure originally described by Selig [12]. The revised procedure is critically examined in section 4.2.4.

\section{Figure 1}

a

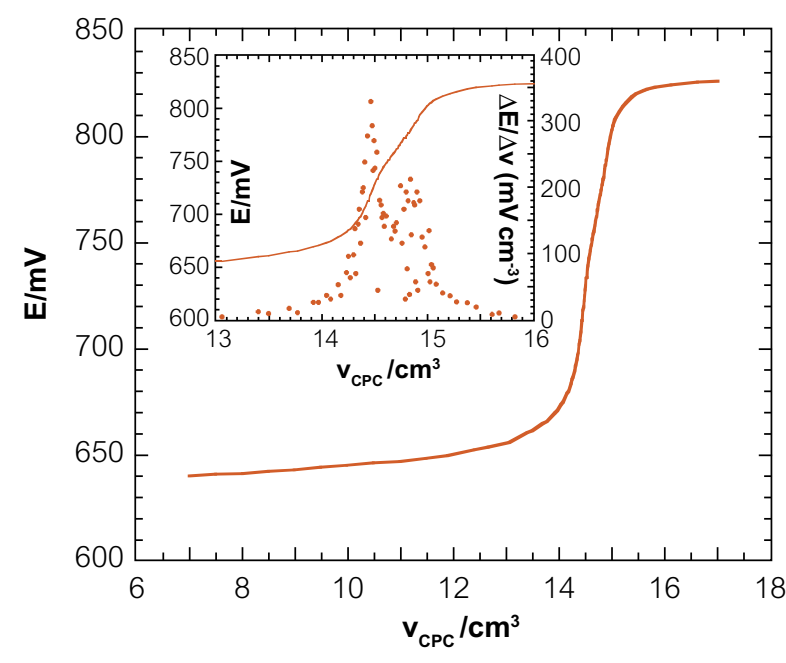

b

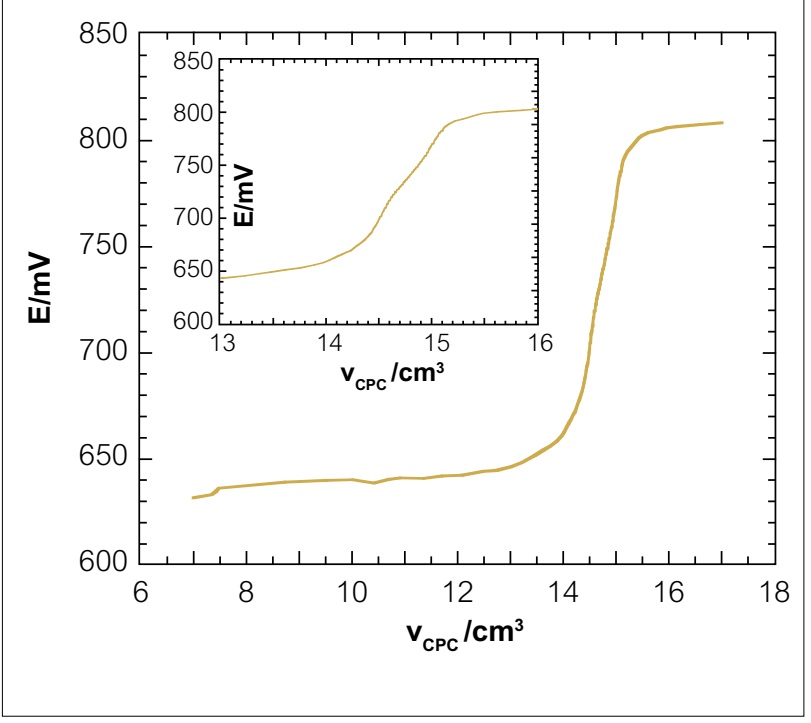

Titration curves of $\mathrm{Au}(\mathrm{III})$ solutions with $5 \cdot 10^{-3} \mathrm{M} \mathrm{CPC}$. Gold solutions are obtained by dissolving pure standard gold (a) and 917/1000 gold alloy (b) respectively. The insets in both figures are magnifications around the equivalent points. The inset in fig. 1a displays also its derivative $E / V_{C P C}$ curve 
The titrations were carried out by a Mettler-Toledo DL-50 automatic titrator equipped with a couple of $20 \mathrm{~cm}^{3}$ burettes. The instrument was computer controlled by $\mathrm{LabX}^{\circledR}$ software, allowing very flexible control of the operations and analysis of the titration data.

\section{Results and discussion}

In this paragraph, the operating procedure will be described and a detailed analysis of every single step, aiming to the evaluation and minimization of the involved errors, will be exploited.

\subsection{Sample dissolution and titration procedure}

An alloy sample, typically containing 150-200 mg of gold exactly weighed, is introduced in the PTFE vial and added to $1.5 \mathrm{~cm}^{3}$ of $\mathrm{HCl}(37 \%)$ and $0.2 \mathrm{~cm}^{3}$ of $\mathrm{HNO}_{3}(67 \%)$. The vial is then located inside the microwave-transparent jacket, screwed and placed in a microwave oven. The sample dissolution is achieved by means of 5 heating cycles. The first two are carried out using a lower power (320w) by previous addition of $\mathrm{HCl}+\mathrm{HNO}_{3}$ mixture, and the last three (400w) are performed after the addition of $1 \mathrm{~cm}^{3}$ of $\mathrm{HCl}(37 \% \mathrm{w})$. These last 3 cycles of heating aim to remove the excess of $\mathrm{NO}_{3}-$ ions via the formation of volatile $\mathrm{NOCl}$. The whole procedure takes about 30 minutes. The solution is then

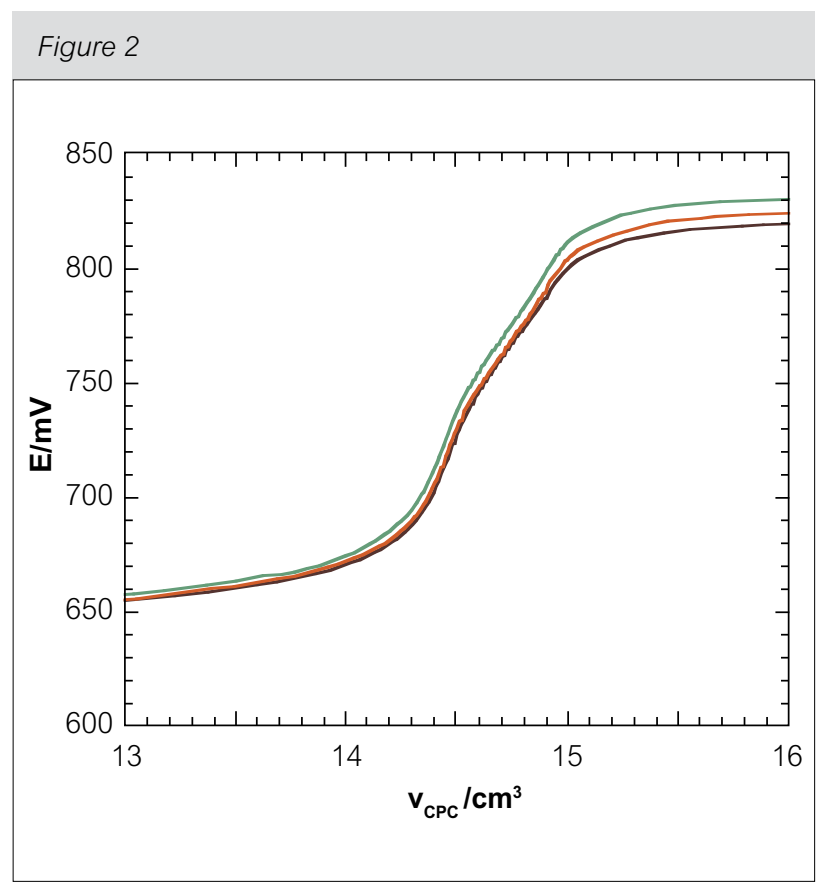

Plots of three consecutive titrations of aliquots of the same pure gold solution quantitatively transferred in a $250 \mathrm{~cm}^{3}$ flask and diluted up to volume by means of $0.1 \mathrm{M} \mathrm{HCl}$. The $\mathrm{pH}$ of the solution so obtained is close to 1 : this value is in the range suggested as optimum for the formation of the gold precipitate [13]. $20 \mathrm{~cm}^{3}$ aliquots of such solution are introduced into the titration vessel by means of a class A+ pipette. The titrating agent is constituted by nearly $5 \cdot 10^{-3} \mathrm{M}$ CPC aqueous solution. The reaction that takes place is the precipitation of the ionic couple according to the following scheme:

$$
\mathrm{AuCl}_{4}^{-}+\mathrm{C}_{21} \mathrm{H}_{38} \mathrm{~N}^{+} \quad \mathrm{C}_{21} \mathrm{H}_{38} \mathrm{~N}^{+} \mathrm{AuCl}_{4}^{-} \downarrow
$$

Since the reaction stoichiometry is $1: 1$, the equivalent point is achievable using about $15 \mathrm{~cm}^{3}$ of solution, a value that can be considered the optimal one when using $20 \mathrm{~cm}^{3}$ volume burettes. Since CPC is characterized by tensioactive properties, it has been found useful to add 1-2 drops of thioether to the stock solution to avoid foam formation. Typical potentiometric titration curves relative to pure gold and gold alloy respectively, are displayed in figure 1 . The curves are characterized by sharp variation of the potential around the equivalent point (about 150$160 \mathrm{mV}$ ). The insets in figure 1 display zoomed views of the curves around this point. The magnification clearly shows the presence of a slight stretching of the rising portion of each curve.

This feature is well evidenced by the derivative curve, where the two regions are highlighted by two well defined peaks (red points in the fig. 1a inset). Even though the exact nature of this double feature has to be determined yet, a large number of experiments provided evidence that the first inflection point is characterized by a more constant and defined shape. Furthermore, its position is in perfect agreement with the reaction stoichiometry. Vice versa, the second one is generally less reproducible (figure 2). Therefore, choosing the first peak is suitable for quantitative analysis purposes.

The automatic titrating machine is equipped with smart data acquisition software $\left(\right.$ LabX $\left.^{\circledR}\right)$ that allows adding the volumes of the titrating solution as a function of the variation of the electrode potential. This feature is capable of increasing the number of points around the equivalent value, where the derivative curves undergo the maximum variation, as well as to speed up the titration where the potential undergoes only slight variations. 


\subsection{Analysis of the procedure precision and accuracy}

In order to optimize the procedure, and to reduce the time required and the errors involved, we subdivided the procedure in 6 single items discussed in the next paragraphs.

\subsubsection{Sampling}

Standard analytical balances normally available in chemical laboratories are characterised by 0.01 mg sensitivity. By weighing about $200 \mathrm{mg}$ of gold (and consequently an even larger sample in the case of alloys) the limited precision of the weighing contributes about $\pm 0.05 \%$ o to the overall error, making this contribution virtually negligible. Vice versa, the sample dissolution turns out to be one of the most important and tricky steps of the procedure. Several attempts were done in order to speed up the sample dissolution and only the modified "aqua regia" procedure satisfies such requirement, allowing the complete dissolution of the sample in no more than 30-40 minutes. A further advantage in using chloride bearing solutions is the formation of an insoluble precipitate of $\mathrm{AgCl}$. Being silver a metal widely used in jewellery alloys, this procedure allows the selective removal of the silver ions from the solution. Since quantitative transfer of solutions is a common laboratory operation, only casual errors can be due to this procedural step when performed by normally skilled operators.

\subsubsection{Standardization of the gold solution}

The tolerance of $250 \mathrm{~cm}^{3}$ class $A+$ volumetric flasks is $\pm 0.6 \%$, while the tolerance of $20 \mathrm{~cm}^{3}$ class $\mathrm{A}+$ pipettes is $\pm 1.5 \%$. The last one is clearly the most critical factor affecting the precision of the whole procedure and, of course, careful volumetric procedures are highly recommended. Repetition of at least 4, or even of more titrations on each sample, aims to partially compensate for the limited precision of pipette: obviously, 4 repetitions lead to halve the relative random error.

\subsubsection{Storage of the titrant solution}

CPC solutions have proved to be stable up to one month, if stored in glass stoppered bottles. Anyhow, it is a good practice to carry out the titrations of the unknown samples of alloy and of the standard gold solution at the same time.

\subsubsection{Preparation and storage of the electrodes}

The best performances of the electrodes are obtained when they are prepared by triplicate immersion of the graphite rods for $10 \mathrm{~s}$ in the surface-modifying
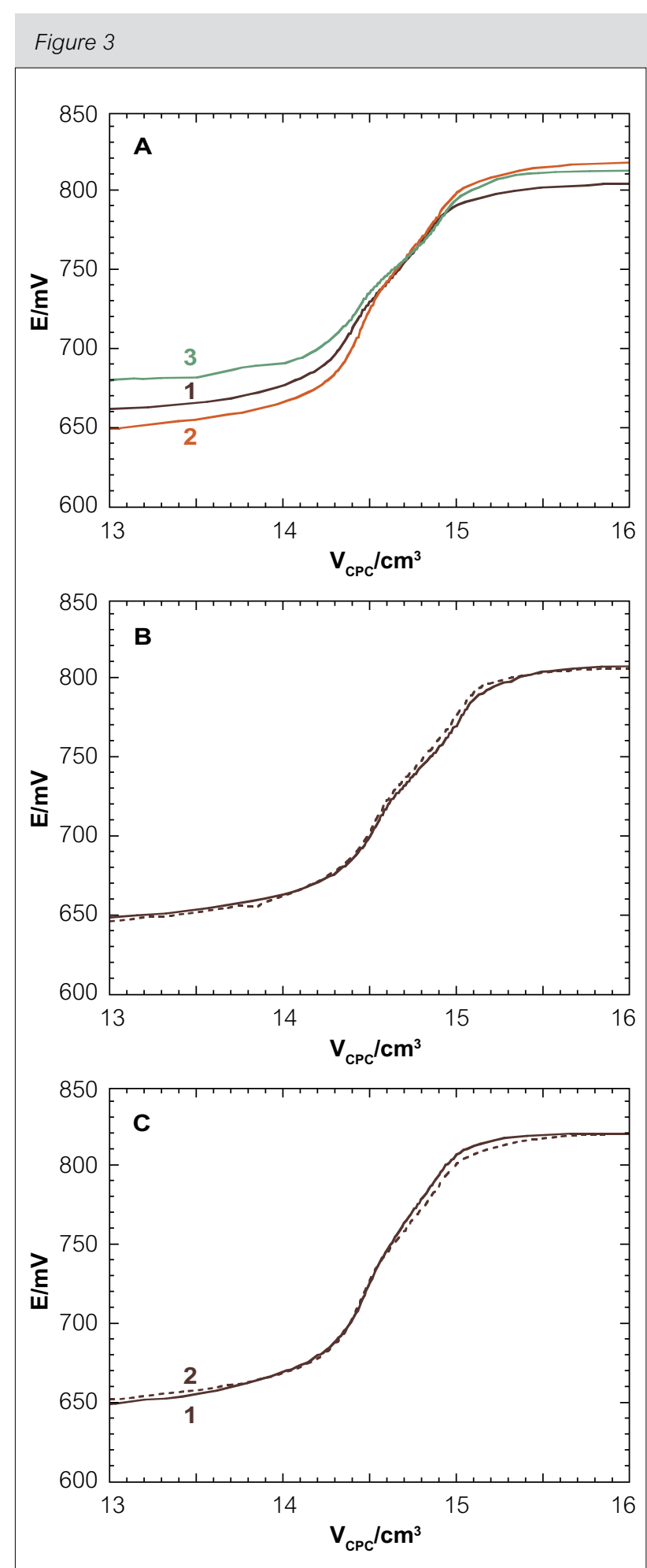

(A) Titration curves of Au(III) with CPC obtained on graphite electrodes prepared by triplicate immersions in DOP-PVC for 3s (blue), 10s (red) and 20s (green), respectively. (B)Titration curves obtained on two different electrodes prepared as in the case of curve A2. (C) Titration curves on one electrode prepared as in A2 and recorded immediately (full line) and after a week (dashed line). 
solution described in the experimental section. The times of immersion must be controlled, since both longer and shorter immersion times give less sensitive electrode surfaces as demonstrated in fig. $3 \mathrm{~A}$. The electrodes prepared in such a way give rise to the best shaped and most reproducible curves, even as far as several days after their preparation (figures $3 \mathrm{~B}$ and $3 \mathrm{C}$ ), by simply storing the electrode in air after rinsing it with distilled water.

\subsubsection{Standardization of the titration parameters}

The software LabX $^{\circledR}$ allows choosing several operative parameters. First we can dispose a maximum titrant volume that is approximately $20 \%$ over the expected equivalent volume; moreover it is possible to predispense about half this volume, without collecting data, to speed up the titration. Then, the key parameters turn out to be the maximum and the minimum volume $\left(\mathbf{v}_{\max }, \mathbf{v}_{\min }\right.$ of progressive titrant addition, and the maximum and the minimum time $\left(\mathbf{t}_{\text {max }}, \mathbf{t}_{\text {min }}\right)$ to wait for obtaining a stable potential after each addition. The values of these parameters have been set up as the optimum ones, after many preliminary tests, as follows:

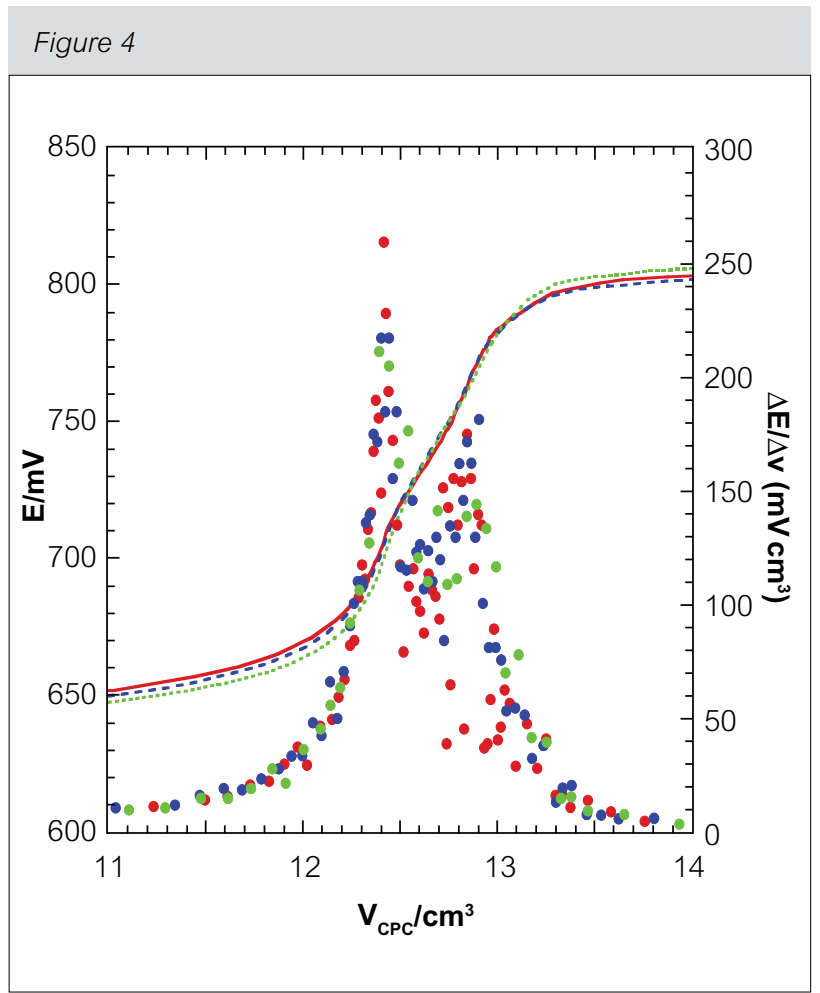

Titration curves (left scale) and corresponding derivatives (points, right scale) relative to $A u(I I I)-C P C$ titrations obtained from the same solutions of both reactants, by setting the minimum additions of titrant at $0.01 \mathrm{~cm}^{3}$ (red), $0.02 \mathrm{~cm}^{3}$ (b/ue) and $0.05 \mathrm{~cm}^{3}$ (green).
- an interval between 2 and $20 \mathrm{~s}$ to wait for stabilization of the signal after each addition of titrant, has proved to be right to assure reproducibility of the results, provided that we make the progressively added titrant volumes as small as possible.

- the variation of the minimum volume of titrant below $0.05 \mathrm{~cm}^{3}$ has a very slight influence on the shape of the derivative curve as shown in fig. 4; anyhow, $\mathbf{v}_{\min }$ has been set at $0.01 \mathrm{~s}$ because a larger number of points $\left(\mathbf{E} / \mathbf{v}_{\mathrm{CPC}}\right)$ around the equivalent point allows a more satisfactory fitting of the derivative curves, as will be discussed in the next paragraph.

\subsubsection{Data elaboration}

The evaluation of the raw experimental data turns out to be a key point since the use of the automatic results provided by the instrument software is not always satisfactory. First of all, the program plots the derivative curve by calculating the incremental ratios (potential over CPC volume, $\Delta \mathbf{E} / \Delta \mathbf{v}$ ) between

\section{Figure 5}

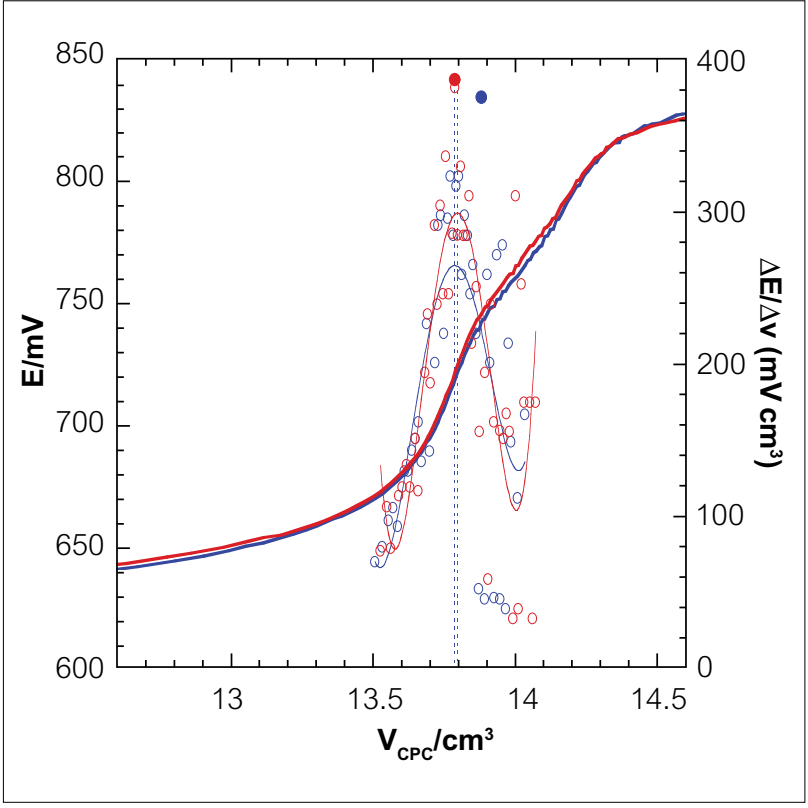

Plots of two consecutive titration curves of $\mathrm{AuCl}_{4}^{-}$with $\mathrm{CPC}$ (thick lines, left scale) and of the corresponding derivative $E / V_{C P C}$ $v s . v_{C P C}$ (points, right scale). Red curve and circles refer to the first titration while blue curve and circles refer to the second one. Only 40 points of each derivative curve are plotted around the equivalent points. Thin lines are the plots of the 8th order polynomial fittings relative to the derivative curves. The full circles are the equivalent points as reported by $L a b X^{\circledR}$ for each of the two curves, while the vertical lines correspond to the maximums of the fittings. 
Table 1. Gold titles of different jewellery alloys obtained by cupellation and potentiometric titration. The data are grouped on the basis of alloy types

\begin{tabular}{|c|c|c|c|}
\hline Type of alloy & $\begin{array}{l}\text { Title \%o dtmn. } \\
\text { by cupellation }\end{array}$ & $\begin{array}{c}\text { Title \% dtmn. by } \\
\text { potentiometric titration }\end{array}$ & $\begin{array}{l}\text { Error \%o towards } \\
\quad \text { cupellation }\end{array}$ \\
\hline $385 / 1000$ & 379,40 & 378.47 & -0.93 \\
\hline $385 / 1000$ & 379,40 & 380.38 & +0.98 \\
\hline $385 / 1000$ & 379,40 & 378.89 & -0.87 \\
\hline $385 / 1000$ & 376,15 & 376.87 & +0.72 \\
\hline $385 / 1000$ & 376,15 & 376.63 & +0.48 \\
\hline $585 / 1000$ & 585,20 & 585,13 & -0.07 \\
\hline $585 / 1000$ & 585,20 & 585.63 & +0.43 \\
\hline $585 / 1000$ & 584,07 & 584.27 & +0.20 \\
\hline $585 / 1000$ & 584,07 & 584.71 & +0.64 \\
\hline $585 / 1000$ & 584.07 & 584.90 & +0.83 \\
\hline $750 / 1000$ & 751.80 & 751.45 & -0.35 \\
\hline $750 / 1000$ & 751.80 & 751.65 & -0.15 \\
\hline $750 / 1000$ & 751.80 & 751.40 & -0.40 \\
\hline $750 / 1000$ & 749.95 & 749.20 & -0.75 \\
\hline $750 / 1000$ & 749.95 & 750.47 & +0.52 \\
\hline $750 / 1000$ & 750.45 & 749.16 & -1.29 \\
\hline $800 / 1000$ & 800.80 & 801.49 & -0.31 \\
\hline $800 / 1000$ & 800.80 & 800.90 & +0.10 \\
\hline $800 / 1000$ & 800.80 & 801.39 & -0.41 \\
\hline $800 / 1000$ & 800.80 & 801.09 & +0.29 \\
\hline $800 / 1000$ & 800.80 & 800.90 & +0.10 \\
\hline $800 / 1000$ & 800.80 & 800.04 & -0.76 \\
\hline $800 / 1000$ & 800.80 & 801.21 & +0.41 \\
\hline $917 / 1000$ & 917.38 & 917.96 & +0.58 \\
\hline $917 / 1000$ & 917.38 & 916.23 & -1.15 \\
\hline $917 / 1000$ & 917.38 & 917.01 & -0.36 \\
\hline $917 / 1000$ & 917.38 & 916.73 & -0.65 \\
\hline
\end{tabular}

two consecutive volumes of titrant and associates them to the second of these volumes. In such a way the graph of the derivative curve is shifted by one half of the added volume. Since the equivalent point volume is typically about $15 \mathrm{~cm}^{3}$ and, around it, the added volumes are $0.01 \mathrm{~cm}^{3}$, the gold titles drawn from each titration are affected by a mean systematic error of approximately $\pm 0.01 /(2 \cdot 15) \cong \pm 0.3 \div 0.4 \%$.

Furthermore, since the software uses the highest $\Delta \mathbf{E} / \Delta \mathbf{v}$ value as criterion for determining the equivalent point, a large number of points collected close to the equivalent point gives sometimes out an automatic result relatively far from the correct one. This is simply due to random errors. In such cases it is useful to fit the derivative curves with polynomial functions in order to reduce the experimental noise and to achieve more reliable results. Figure 5 helps to clarify this key point. Here an expanded view of the equivalent-point zone of two consecutive titration curves is displayed. The thick lines represent the electrode potential values as function of the added 
CPC volumes, while the circles represent their $\Delta \mathbf{E} / \Delta \mathbf{v}$ values respectively (for clarity only 40 points are plotted). In particular the red curve and circles refer to the first titration, while the blue curve and circles refer to the second titration. Moreover, the full circles represent the two equivalent points as automatically determined by the software. It is evident that there is a significant difference (about 7\%o) between these two points. However, if both derivative curves are fitted by means of 8th order polynomial curves (thin lines in figure 5), the abscissae of their maximums are almost coincidental (difference $0.7 \%$ ). The increase of precision of about one order of magnitude as illustrated by this example is typically verified in a large number of experiments.

\subsection{Test on different types of goldsmithery alloys}

In order to evaluate the method suitability as standard procedure for the determination of the gold in real jewellery samples, we analyzed a large number of different types of alloys currently used for goldsmithery purposes. The alloys, whose nominal composition was ranging from 385/1000 up to $917 / 1000$, were granted from commercial firms together with their gold titles carefully determined by cupellation. Such certified values were taken as "true" and used as a benchmark for the potentiometric data. The results are summarized in table I. The differences between potentiometric titrations and cupellation are displayed in column 4 and, with only a couple of exceptions, are below the limit of $1 \%$. The mean error of the gold content of all samples found by potentiometric titration towards cupellation is $-0.08 \%$, while the relative standard deviation of the errors is $\pm 0.6 \%$, providing evidence of the accuracy and versatility of the potentiometric method.

\section{Conclusions}

An experimental method forquantitative determination of gold in jewellery alloys by potentiometric titration has been thoroughly investigated. This work started from the pioneering studies by Selig [11-13] and is based upon the precipitation of $\mathrm{AuCl}_{4}^{-}$with cethylpyridinium cation. All steps involved in the whole procedure have been revised and optimized to set up a complete analytical pathway as well as to get the best level of accuracy. The present method can be claimed to be of practical interest, even though it does not reach the accuracy assured by cupellation; in particular it offers several advantages:
- It consists on simple procedures.

- The cost of the equipment and of the consumables is by far negligible in comparison with the huge apparatus and waste of energy characterizing the cupellation.

- The use of large amounts of lead, that issues a big problem of toxic wastes, is not involved.

- The amount of precious metal needed by the potentiometric method is less than half the one necessary for cupellation.

While we are developing new promising experiments on a commercial electrode with the same titration approach, we can conclude that, taking into account the numerous advantages listed above, the results obtained with the simple surface-modified graphite electrode allow to set the potentiometric procedure here described as an alternative, or at least a complementary, analytical method to cupellation.

\section{Acknowledgements}

We are grateful to Dr. Mauro Caneschi from Sagor (Arezzo, Italy), for the cupellation tests and the helpful advice and discussion on the jewellery alloys. Thanks are also due to Mettler Toledo for equipment grant.

\section{About the authors}

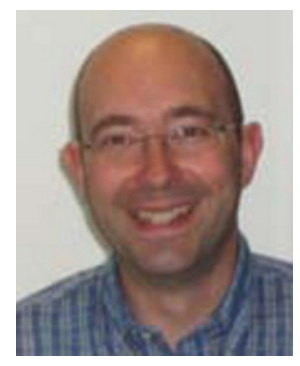

Stefano Caporali graduated in Chemistry in 1998 and received his Ph.D degree in Materials Science from University of Florence (Italy) in 2006. His research interests cover a wide range of applied and theoretical aspects of electrochemistry and surface chemistry in both aqueous and non-aqueous environments.

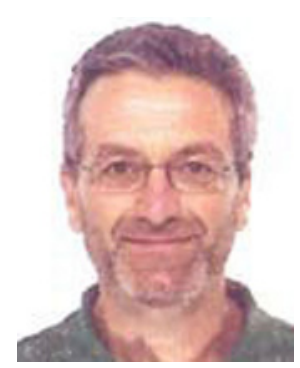

Silvano Bellandi graduated in Biology at the University of Florence in 1980, since 1987 he works in Analytical Strumental Chemistry and since 2005 in Electrochemistry with regard to nanomaterials and methods for determination of trace metals. 


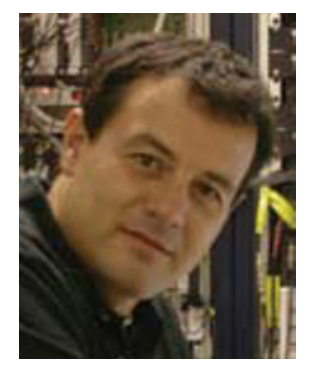

Massimo Innocenti graduated in Chemistry at the University of Florence in 1989; since November 2002, he is researcher in ElectroAnalytical Chemistry. The scientific activity concerned different topics, all of them in the field of Electrochemistry, with a particular attention to the electrodeposition of metals and semiconductors under morphological control.

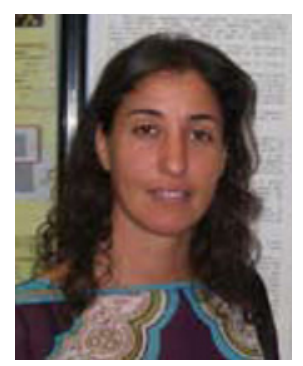

Ornella Lopilato graduated in Chemistry at the University of Florence in 2007. She is working in ElectroAnalytical Chemistry and her scientific activity concerned the use of electrochemical techniques for the determination of environmental pollutant.

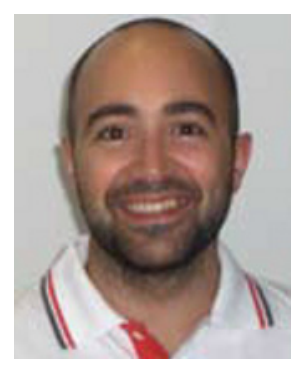

Lorenzo Romualdi graduated in Chemistry at the University of Florence in 2010. His scientific activity concerned the use of electrochemical techniques for the determination of precious metals.

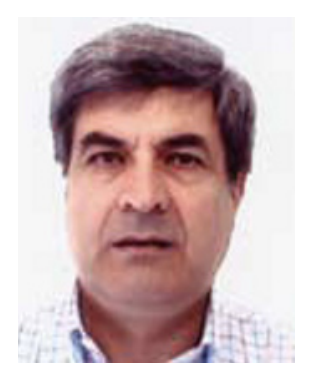

Giovanni Pezzatini is associate professor of Analytical Chemistry in the University of Florence. Since 1982 he was teaching different analytical subjects in Florence and Siena Universities. He is the author of 70 research papers in international journals, concerning electroanalytical chemistry. This last is the subject in which he is now encharged in the department of chemistry of Florence University.

\section{References}

1 C.W. Corti, Gold Technology 2001, 32, 20

2 F.E. Beamish, The Analytical Chemistry of the Noble Metals, Pergamon, Oxford, 1966

3 UNI EN ISO 11426, Determination of gold in gold jewellery alloys, 2000

4 M. Brill, K.H. Wiedemannl, Gold Bull. 1992, 25, 13

5 J.C. Van Loon, R.R. Barefoot, Determination of the precious metals, Wiley, Chichester, 1991, and references therein

6 P. Jalas, J.P. Ruottinen, S. Hemminki, Gold Technology 2002, 35, 28

7 M. Corsi, G. Cristoforetti, V. Palleschi, A. Salvetti, E. Rognoni, Eur. Phys. J., 2001, D 13, 373

8 V. Roessiger, B. Nensel, Gold Bull. 2003, 36, 125

9 A. Jurado-Lopez, M.D. Luque De Castro, Spectrochim. Acta 2003, Part B 58, 1291

10 U. Bardi, F. Niccolai, M. Tosti, A. Tolstogouzov, Int. Journal Mass Spectr. 2008, 273, 138

11 W.S. Selig, Fresenius Z. Anal. Chem. 1982, 312, 419, and references therein

12 W.S. Selig, J. Chem. Ed. 1984, 61, 80

13 W.S. Selig, Anal. Bioanal. Chem. 1985, 320, 562, and references therein

14 L.C. Robbles, C. Garcia-Olalla, A.J. Aller, Fresenius J. Anal. Chem. 1993, 345, 441

15 K. Someya, Z. Anorg. Chem. 1930, 187, 337

16 W.B. Pollard, Analyst 1937, 62, 597

17 F.F. Beamish, J.J. Russell, J. Seath, Ind. and Engineering Chem. 1937, 9, 475

18 S.C. Soundar Rajan, N. Appala Raju, Talanta 1975, 22, 185

19 http://www.parrinst.com/doc_library/members/4780MW.pdf

20 U.S. Patent No. 4882128, Pressure and temperature reaction vessel, method, and apparatus, 1989, November 21 th 